\title{
Les adolescents qui présentent des troubles du comportement sexuel et leurs parents : en quoi se distinguent ceux dont la situation n'est pas judiciarisée ? \\ Adolescents who have sexual behavior problems and their parents: how do those whose situation is not adjudicated differ?
}

\author{
Denis Lafortune, Dominique Meilleur et Janick Coutu
}

Volume 42, numéro 1, 2013

URI : https://id.erudit.org/iderudit/1061725ar

DOI : https://doi.org/10.7202/1061725ar

Aller au sommaire du numéro

Éditeur(s)

Revue de Psychoéducation

ISSN

1713-1782 (imprimé)

2371-6053 (numérique)

Découvrir la revue

Citer cet article

Lafortune, D., Meilleur, D. \& Coutu, J. (2013). Les adolescents qui présentent des troubles du comportement sexuel et leurs parents : en quoi se distinguent ceux dont la situation n'est pas judiciarisée ? Revue de psychoéducation, 42(1), 95-114. https://doi.org/10.7202/1061725ar

\section{Résumé de l'article}

Les adolescents qui manifestent des troubles du comportement sexuel ne sont pas tous pris en charge par le système de justice pénale pour les adolescents. En effet, un certain nombre d'entre eux consultent dans un milieu médical ou reçoivent des services en vertu de la Loi sur la Protection de la Jeunesse. L'objectif de cette étude est de vérifier s'il existe des différences entre les adolescents judiciarisés et non judiciarisés. L'échantillon est composé de 30 jeunes auteurs d'agression sexuelle ayant consulté en clinique médicale générale ou spécialisée ainsi que 17 adolescents ayant des gestes comparables et pris en charge en vertu de la Loi sur le Système de Justice Pénale pour Adolescents. Leurs parents ont également été sollicités. Parmi ceux-ci, 20 sont des parents d'adolescents non judiciarisés et six des parents de jeunes judiciarisés. Dans l'ensemble, les jeunes des deux groupes qui ont participé à l'étude ne rapportent que peu de distorsions cognitives, attributions externes du blâme, valeurs délinquantes et conflits ouverts avec leurs parents. Ceux qui n’ont pas été judiciarisés rapportent significativement plus de symptômes dépressifs, anxieux et dissociatifs. La plupart de ces adolescents ont consulté antérieurement dans un ou plusieurs autres services. Quant aux parents, ils se distinguent très peu d'un groupe à l'autre. Tous rapportent vivre de fréquents conflits intrafamiliaux, mais peu croient avoir des problèmes majeurs sur le plan des pratiques éducatives.
Tous droits réservés $@$ La Corporation de la Revue Canadienne de Psycho-Éducation, 2013
Ce document est protégé par la loi sur le droit d'auteur. L'utilisation des services d'Érudit (y compris la reproduction) est assujettie à sa politique d'utilisation que vous pouvez consulter en ligne. 


\section{Les adolescents qui présentent des troubles du comportement sexuel et leurs parents : en quoi se dis- tinguent ceux dont la situation n'est pas judiciarisée?}

\section{Adolescents who have sexual behavior problems and their parents: how do those whose situation is not adjudicated differ?}

\section{Lafortune ${ }^{1}$ \\ D. Meilleur ${ }^{2}$ \\ J. Coutu ${ }^{2}$}

1 École de criminologie, Université de Montréal

2 Département de psychologie, Université de Montréal

\section{Correspondance :}

Denis Lafortune, professeur titulaire

École de criminologie, Université de Montréal

C.P. 6128 , succursale centre ville Montréal, QC H3C 3J7

\section{Résumé}

Les adolescents qui manifestent des troubles $d u$ comportement sexuel ne sont pas tous pris en charge par le système de justice pénale pour les adolescents. En effet, un certain nombre d'entre eux consultent dans un milieu médical ou reçoivent des services en vertu de la Loi sur la Protection de la Jeunesse. L'objectif de cette étude est de vérifier s'il existe des différences entre les adolescents judiciarisés et non judiciarisés. L'échantillon est composé de 30 jeunes auteurs d'agression sexuelle ayant consulté en clinique médicale générale ou spécialisée ainsi que 17 adolescents ayant des gestes comparables et pris en charge en vertu de la Loi sur le Système de Justice Pénale pour Adolescents. Leurs parents ont également été sollicités. Parmi ceux-ci, 20 sont des parents d'adolescents non judiciarisés et six des parents de jeunes judiciarisés. Dans l'ensemble, les jeunes des deux groupes qui ont participé à l'étude ne rapportent que peu de distorsions cognitives, attributions externes du blâme, valeurs délinquantes et conflits ouverts avec leurs parents. Ceux qui n'ont pas été judiciarisés rapportent significativement plus de symptômes dépressifs, anxieux et dissociatifs. La plupart de ces adolescents ont consulté antérieurement dans un ou plusieurs autres services. Quant aux parents, ils se distinguent très peu d'un groupe à l'autre. Tous rapportent vivre de fréquents conflits intrafamiliaux, mais peu croient avoir des problèmes majeurs sur le plan des pratiques éducatives.

Mots-clés : adolescents - agression sexuelle - parents judiciarisation - cliniques médicales

\section{Abstract}

Adolescents who commit reprehensible acts of a sexual nature do not all appear before the juvenile courts. A certain number of them are seen by a medical specialist or receive services under the Youth Protection Act. The objective of this study is to verify whether or not there exist differences on a number of points between adolescents who appear before the courts and those who do not. The sample is 
made up of 30 adolescent sex offenders who were seen in a specialized or family medical clinic as well as 17 adolescents who committed the same kind of act, but who came under the Youth Criminal Justice Act. Parents were also asked to participate. Among those who volunteered, 20 are parents of adolescents who did not appear before the courts and six are parents of adolescents who did. Overall, the youth who participated in the study reported hardly any cognitive distortions, external attributions of blame, delinquent values or open conflicts with their parents. Those who received medical services and were not adjudicated have significantly more symptoms of depression, anxiety and dissociative disorders. Most of these adolescents have been seen in the past by one or more services. With respect to the parents, there is very little to distinguish between the two groups. All reported experiencing frequent conflicts within the family, but do not believe they have any major problems in terms of educative practices.

Key words: adolescents - sexual aggression - parents - adjudication - medical clinic

\section{Introduction}

L'hypothèse de l'expérimentation soutient que certains adolescents auteurs d'agression sexuelle n'ont que commis une erreur de parcours. Depuis longtemps, certains parents, intervenants et avocats sont réticents à judiciariser la situation, craignant que l'étiquette d'agresseur sexuel ne porte ensuite préjudice au jeune. Par exemple, il y a vingt ans, Gaudreau (1991) affirmait que l'abus sexuel commis par un adolescent peut s'expliquer par des phénomènes relativement normaux, tels que l'excitation sexuelle, la curiosité et l'accès non supervisé à des enfants. Depuis tout aussi longtemps, cette thèse est décriée par d'autres auteurs, qui la jugent surprotectrice. II y a trente ans déjà, Longo et Groth (1983) ont déploré le fait que plusieurs délinquants adultes avaient été repérés plus jeunes, mais que leur situation avait été minimisée et qualifiée de simples troubles de l'adaptation ou troubles du comportement. C'est ainsi que ce sont progressivement mises en place deux grandes stratégies d'intervention auprès des jeunes : avec ou sans judiciarisation de l'acte sexuel problématique.

Au Québec, la même situation prévaut. Certains comportements sexuels abusifs ou agressifs sont évalués et, par la suite, pris en charge par des travailleurs sociaux, psychologues, médecins généralistes ou pédopsychiatres dans un contexte de non-judiciarisation (Lafortune, 2009). Plusieurs de ces consultations hors du système de justice pénale sont engagées dans des cliniques médicales ou pédopsychiatriques. Parfois, une évaluation et un suivi sont assurés par des intervenants qui ne sont pas nécessairement spécialistes en la matière (ex. : une clinique de pédiatrie ou de pédopsychiatrie générale). D'autres fois, un programme spécifique pour les " troubles sexuels " a été mis en place (ex. : une intervention individuelle et de groupe offert dans un département de pédopsychiatrie légale). Les premiers organismes peuvent donc être qualifiés de généralistes et les seconds de spécialisés. Enfin, les jeunes peuvent aussi faire face à des procédures légales et être accusés d'agression sexuelle.

Au plan scientifique, il peut être utile de rappeler que l'agression sexuelle juvénile n'intéresse pas que le champ pénal. Au cours des années ' 70 et ' 80 , les premières études empiriques portant sur les garçons sexuellement agressifs ont 
été publiées dans de revues de pédopsychiatrie, telles que Journal of the American Academy of Child and Adolescent Psychiatry (Kavoussi, Kaplan, \& Becker, 1988), ou des revues de service social et de psychologie, telles que Journal of Family Violence (Kaplan, Becker, \& Cunningham-Rathner, 1988). Depuis, la majorité des publications reposent sur des échantillons variés, regroupant des jeunes de centres de réadaptation, prisons pour mineurs, organismes communautaires et cliniques spécialisées en délinquance sexuelle (Tardif, Jacob, Quenneville, \& Proulx, 2012).

À notre connaissance, aucune étude ne s'est encore penchée spécifiquement sur un sous-groupe d'adolescents consultant en marge du système de justice pénale pour les adolescents. Dans cette étude, par trouble du comportement sexuel (ci-après TCptSx), il faut entendre des comportements qui n'ont pas été judiciarisés en vertu de la Loi sur le Système de Justice pour les Adolescents (ci-après LSJPA). Un bon nombre de ces gestes peuvent néanmoins avoir fait l'objet d'un signalement en vertu de la Loi sur la Protection de la Jeunesse (ci-après LPJ). Autrement dit, l'expression est réservée aux jeunes non judiciarisés, tandis que celle d'adolescent auteur d'agression sexuelle (ci-après AAAS) désigne ici tous les participants, qu'ils aient été judiciarisés ou non. Dans les lignes qui suivent, nous décrivons les caractéristiques des AAAS les plus souvent étudiées, aux plans individuel et familial.

Caractéristiques individuelles des AAAS. De manière générale, malgré le lien étroit qu'ils peuvent entretenir avec leur victime (ex. : une sœur ou un cousin), les AAAS expriment assez peu d'empathie et de remords face aux gestes commis lorsqu'on les interroge (Kaplan, Becker, \& Martinez, 1990; Saferbi, 1990). En effet, des distorsions cognitives et une minimisation du sentiment de responsabilité teintent souvent la réaction qu'ont ces adolescents après leur acte (Bengis, 1997). Malheureusement, il est impossible de savoir si ces caractéristiques varient selon que la situation du jeune ait été judiciarisée ou non.

En outre, dans l'histoire de plusieurs AAAS, on retrouve d'autres troubles du comportement et signes de mésadaptation sociale. Selon les auteurs québécois qui ont analysé la question, de 44\% (Jacob et al., 1993) à 73\% (Madrigrano et al., 1997) des AAAS ont des antécédents de délinquance non sexuelle, officiels ou non. Ces résultats suggèrent une trajectoire polymorphe, caractérisée à la fois par le vandalisme, les méfaits, les agressions physiques et les petits vols (Carpentier, Leclerc, \& Proulx, 2011; LeBlanc \& Lapointe, 1999). L'Inventaire de Jesness (1983) est un instrument conçu expressément pour évaluer les traits de personnalité et la mésadaptation sociale des délinquants juvéniles. Les AAAS regroupés par Dozois (1994) y obtiennent un score supérieur au seuil clinique $(T \geq 60)$ sur l'échelle de mésadaptation sociale et l'indice d'asocialité. Ce résultat a été reproduit par Lafortune (1996; scores pondérés moyens de 62 à l'échelle de mésadaptation sociale et 61,3 à l'indice d'asocialité), et par LeBlanc et Lapointe (1999, 64,7 pour l'échelle mésadaptation sociale). Cela dit, selon ces auteurs, aucune échelle du Jesness ne parvient à distinguer clairement les AAAS des autres délinquants juvéniles, ce qui montre l'importance de considérer des éléments plus spécifiques à la sexualité. 
Plusieurs travaux ont traité d'un possible cycle de la violence sexuelle et de la présence de symptômes de stress post-traumatiques chez les AAAS (Rightland \& Welch, 2001). Parmi les garçons de la population générale, la prévalence des agressions sexuelles vécues se situe entre $7 \%$ et $9 \%$. Chez les AAAS, ce taux s'élève à près de 30\% (Hanson \& Slater, 1999; Lagueux \& Tourigny, 1999; Lafortune 2001). Si cet antécédent biographique est fréquent, son rôle dans l'étiologie du trouble reste obscur puisque, de toute évidence, toutes les victimes masculines ne posent pas de gestes agressifs par la suite. Le passage de la position de victime à celle d'agresseur doit donc provenir de la conjonction de plusieurs vulnérabilités, dont certaines relèvent de la dynamique familiale. Vizard, Hickey, French et McCrory (2007) ont décrit des AAAS consultant dans un centre de traitement spécialisé situé au Royaume-Uni et souligné le fait qu'ils ont vécu plusieurs types d'abus durant leur enfance. En effet, $71 \%$ des AAAS de l'échantillon ont été victimes d'abus sexuels, $66 \%$ d'entre eux ont été abusés physiquement et $74 \%$ ont subi des abus émotionnels et psychologiques. Une proportion significative de ces adolescents a été retirée de leur famille par les services sociaux (76\%) pour protéger leur sécurité et leur développement.

Caractéristiques du milieu familial. Qu'ils adoptent une approche développementale, systémique ou cognitivo comportementale, plusieurs chercheurs et cliniciens ont souligné l'importance de l'environnement familial dans l'étiologie de l'agression sexuelle (Born \& Thys, 2001; Loeber \& Farrington, 2000). Dans plusieurs familles d'AAAS, on constate d'abord qu'un des parents a vécu de la maltraitance en bas âge. Selon les études américaines, de 21\% (Kaplan et al., 1988) à 67\% (Johnson, 1989) de ces adolescents ont un parent ou grand-parent qui a été victime d'agression sexuelle durant sa propre enfance. L'histoire traumatique se retrouve souvent chez les mères (Knopp, 1985), ce qui peut entraîner à l'âge adulte, de la détresse et des difficultés à évaluer les besoins de l'enfant et encadrer les écarts de conduite.

Dans une recension des écrits, Vizard, Monck et Misch (1995) indiquent que la majorité des familles d'AAAS éprouvent des dysfonctionnements notables. Parmi ceux-ci, l'adaptabilité désigne la capacité d'une famille à modifier sa structure, les rôles de chacun, ainsi que ses règles afin de s'ajuster aux différentes situations. Or, cette capacité serait réduite chez les familles d'AAAS. Busconi, Gray, Houchens et Pithers (1998) y observent également des relations d'attachement plus insécurisées. Enfin, ces familles tendent à présenter plus de conflits que la moyenne (Ford \& Linney, 1995).

Sous l'angle des pratiques éducatives, Worling (1995) a remarqué que les AAAS subissent plus de punitions corporelles que les autres adolescents. Blaske, Borduin, Henggeler, et Mann (1989) mentionnent que l'absence de soutien et de supervision de la part des parents peut être souvent constatée dans les familles où un jeune a commis une agression sexuelle. Selon Vizard, Hickey, French et McCrory (2007), la discipline parentale inconsistante (64\%) ou absente $(49 \%)$ est fréquente dans l'histoire de vie des AAAS.

La notion d'empowerment ou pouvoir d'agir (Dufort \& Lebossé, 1999) désigne la capacité d'une personne à mobiliser ses forces et ressources pour 
affronter une épreuve. Dans le domaine qui nous intéresse, dès 1991, Thomas a mentionné l'importance pour les parents de rester en contrôle lors des prises de décisions et d'actions qui suivent le dévoilement d'une agression sexuelle. À l'aide du Parenting stress index, Busconi (et al., 1998) ont observé que les parents ayant un AAAS ont tendance à vivre plus de stress et à se sentir plus inadéquats vis-à-vis leurs tâches et leur rôle. Pour les intervenants, ce sentiment d'être dépassés par les événements (ex. : être bouleversé et incapable de prendre sa place) doit être pris en compte dans la planification du suivi.

Bref, au fil des ans, le portrait des AAAS et de leurs parents s'est précisé. Toutefois, on n'en sait que très peu sur la spécificité des adolescents qui manifestent des TCptSx et qui restent à l'écart du système de justice pénale. À notre connaissance, les études qui s'approchent le plus de cette question l'abordent sous l'angle de la comparaison entre AAAS et autres délinquants juvéniles, ce qui reste une approche très indirecte.

Ainsi, Jonson-Reid et Way (2001) n'ont pas trouvé de différences entre un groupe d'AAAS et un groupe de délinquants juvéniles quant à leurs antécédents d'abus sexuels et physiques. Par contre, les AAAS étaient plus nombreux à rapporter diverses formes de maltraitance tandis que les autres jeunes délinquants étaient plus à risque de n'avoir été que victimes de négligence. De plus, les AAAS se distinguaient des autres délinquants juvéniles par leurs antécédents, étant plus susceptibles d'avoir été condamnés à trois reprises ou plus dans le passé. Quant à Burton, Millet et Shill (2002), ils se sont intéressés aux antécédents de victimisation sexuelle chez des AAAS et des délinquants juvéniles non sexuels. Ils ont observé que les premiers étaient plus susceptibles d'avoir été agressés par une personne proche de leur entourage, que leur agresseur était plus souvent de sexe masculin et que leur historique d'abus était plus long.

Cette étude se penche donc sur les caractéristiques distinctives des AAAS dont la situation n'est pas judiciarisée. Elle poursuit deux objectifs : a) dresser le portrait d'un échantillon de jeunes qui manifestent des TCptSx et consultent en milieu médical pour ensuite le comparer à celui d'adolescents judiciarisés et suivis en vertu de la LSJPA et b) reprendre ce parallèle au niveau des caractéristiques familiales.

\section{Méthode}

\section{Participants}

L'étude, d'une durée de 18 mois, a été réalisée en collaboration avec 15 organismes québécois : sept cliniques médicales ou de pédopsychiatrie générale (ex. : Centre des Adolescents du Pavillon Albert Prévost), trois cliniques pédopsychiatriques spécialisées (ex. : Centre hospitalier Robert-Giffard) et cinq milieux accueillant des jeunes judiciarisés (ex. : Centre d'intervention en délinquance sexuelle de Laval). Une subvention ${ }^{1}$ accordée par le Centre national de prévention du crime et le ministère de la Sécurité publique a permis sa réalisation.

1. Projet CNPC 3150- C7, devenu 3150-U3 
Les 47 adolescents recrutés ont reconnu soit a) avoir agressé sexuellement une adolescente ou b) avoir eu des contacts sexuels avec un enfant avec qui ils avaient au moins trois ans d'écart d'âge. Ces comportements peuvent s'être manifestés dans un contexte intra- ou extra-familial. Le premier groupe est composé de 30 jeunes non judiciarisés. De ceux-là, sept ont consulté dans des cliniques médicales générales et 23 dans des cliniques spécialisées. Pour les recruter, il a fallu approcher 39 jeunes (taux de réponse de 77\%). Ils ont été signalés en vertu de la Loi sur la Protection de la Jeunesse $(n=21)$ ou ne sont sous la juridiction d'aucune loi $(n=9)$. Le deuxième groupe est composé de 17 jeunes judiciarisés. Ils ont été recrutés dans cinq milieux d'intervention psychosociale, de manière à constituer un groupe témoin. Dans ce cas, 21 jeunes ont initialement été pressentis (taux de réponse de $81 \%$ ).

Par « parent », il faut entendre ici un parent biologique, par alliance ou adoptif qui est désigné par un jeune participant comme étant une personne significative. Au total, 66 parents ont été approchés, mais le taux de refus a été assez important (60\%). Les 26 parents volontaires se répartissent en deux groupes : 20 fréquentent des cliniques médicales générales ou spécialisées, leur fils étant suivi en vertu de la LPJ. Les six autres proviennent des milieux d'intervention psychosociale, leur fils étant judiciarisé et encadré par la LSJPA. Ils ont été recrutés afin de constituer un groupe témoin. La mère biologique a rempli les questionnaires dans $66 \%$ des cas (14 des 20 en clinique médicale et 4 des 6 en milieu d'intervention psychosociale); les autres l'ont été par le père biologique.

L'étude a été approuvée par les comités d'éthique à la recherche de la Faculté des Arts et des Sciences de l'Université de Montréal et du Centre hospitalier de Québec (reconnu par le Ministère de la Santé et des Services sociaux). Tous les jeunes participants de même que leurs parents ont signé un formulaire attestant de leur consentement éclairé. La collecte de données a été réalisée lors des cinq premières semaines de la prise en charge des adolescents.

\section{Instruments}

Six instruments et quelques questions relatives au statut socioéconomique ont été administrés aux jeunes.

Le questionnaire portant sur les distorsions cognitives des adolescents (Becker \& Kaplan, 1988; 32 items) mesure les rationalisations et distorsions cognitives. À chaque item, le jeune doit répondre par vrai ou faux, le résultat brut étant le total de "vrais ». À défaut de score $\mathrm{T}$, une division du score brut par le maximum possible de 32 peut le convertir en un indice décimal variant entre 0 et 1. Selon Madrigrano, Robinson et Rouleau (1997), les coefficients de consistance interne de la version française sont satisfaisants $(\alpha=0,45-0,71)$.

L'échelle d'attribution de la responsabilité (Gudjonsson \& Singh, 1989; 42 items) regroupe trois sous-échelles : sentiment de culpabilité, attribution de responsabilité externe et attribution à un état mental perturbé. Pour chacun des items, le participant doit répondre vrai ou faux. Les résultats bruts sont les totaux de « vrais " pour chacune des sous-échelles. À défaut de scores T, une division par le 
maximum possible (18 pour la sous-échelle de culpabilité, 15 pour les attributions externes et 9 pour les attributions à un état mental perturbé) permet de convertir en indices décimaux variant entre 0 et 1 les scores bruts. Pour chacune des trois sous-échelles, les indices de consistance interne varient de 0,72 à 0,80 .

L'inventaire de personnalité de Jesness (1983, 155 items) a été conçu et validé en Californie. Les énoncés, auxquels le participant répond par vrai ou faux, sont regroupés en dix échelles et un indice synthèse. Les trois premières échelles, mésadaptation sociale, orientation des valeurs et immaturité, ont été construites à partir d'un cadre conceptuel. Les sept autres (autisme, aliénation, agressivité manifeste, retrait, anxiété sociale, refoulement et déni) ont été construites suite à une analyse factorielle. Quant à l'indice global d'asocialité, il est un bon indicateur de la mésadaptation sociale. Les données brutes obtenues sur onze dimensions peuvent être converties en scores $T$ au moyen d'une table, le seuil clinique étant établi à 60. Les analyses de la qualité psychométrique indiquent des coefficients de fiabilité Odd-Even allant de 0,62 à 0,88 , ainsi qu'une stabilité test-retest variant entre 0,40 et 0,70 (Jesness, 1983).

L'échelle des symptômes traumatiques pour enfants (Brière, 1989; 54 items) est une adaptation française abrégée du "Trauma Symptom Checklist for Children " (TSC-C). Chacun des items est suivi d'une échelle Likert en quatre points. Six échelles cliniques mesurent la perception de l'adolescent quant à son niveau d'anxiété, dépression, stress post-traumatique, préoccupations sexuelles, dissociation et colère. Deux indices de validité évaluent la tendance des adolescents à minimiser la présence de symptômes et la tendance, au contraire, à l'exagérer. Les données brutes peuvent être converties en scores $\mathrm{T}$, le seuil clinique étant fixé à 60 . Pour la version française, les indices de consistance interne varient de 0,70 à 0,84 (Lagueux, 2006).

Les échelles d'attitudes face au père (Guili \& Hudson, 1977; 25 items) et d'attitudes face à la mère (Guili et Hudson, 1977; 25 items) sont les adaptations françaises du Child Attitude towards Mother et du Child Attitude towards Father (Comeau \& Boisvert, 1985). Elles permettent d'évaluer la qualité perçue de la relation avec les parents. Les items sont suivis d'une échelle Likert en 5 points. Plus le résultat est bas, meilleure est la qualité perçue de la relation. À défaut de score $\mathrm{T}$, une division du total par le nombre d'items permet d'identifier la réponse la plus souvent choisie sur l'échelle. L'indice de cohérence interne de la version française a été évalué à 0,90 (Pauzé, Centre Jeunesse de Montréal, \& Yergeau, 2004).

Enfin, un indice de niveau socioéconomique a été calculé à partir de l'âge du parent le plus significatif pour le jeune, son niveau de formation et sa catégorie professionnelle, selon la méthode proposée par Genoud (2011), à savoir Indice = âge - (6x Niveau de Formation) - (4 x Catégorie professionnelle) +55 . En principe, le résultat varie entre 1 et plus de $80^{2}$.

2. Pour plus de détails, consulter http://www.unifr.ch/ipg/assets/files/DocGenoud/IPSE_new.pdf 
L'évaluation des caractéristiques familiales repose sur les cinq instruments suivants.

La trajectoire de consultations antérieures à la demande actuelle (Pauzé, Centre Jeunesse de Montréal, \& Yergeau, 2004; 10 items) porte sur les consultations faites pour le jeune auprès de ressources et d'organismes. Le parent doit identifier les services antérieurs qu'il a déjà utilisés pour un problème émotionnel ou comportemental de son adolescent. Pour chaque service utilisé, des détails doivent être fournis. Cet outil n'est pas constitué d'échelles et ne se prête donc pas à des études de fidélité ou validité.

Le questionnaire portant sur les souvenirs traumatiques de l'enfance (Bernstein, Fink, Handelsman, Foote et al., 1994; Paivio \& Cramer, 2004; 70 items) mesure la fréquence de certains souvenirs de mauvais traitements vécus dans l'enfance du répondant. Les items, suivis d'une échelle Likert en cinq points, concernent : l'abus physique et émotionnel (23 items), la négligence émotionnelle (16 items), la négligence physique (11 items) et l'abus sexuel (6 items). À défaut de score $\mathrm{T}$, une division du score brut par le nombre d'items permet d'identifier la réponse la plus choisie sur l'échelle. Selon Paquette, Laporte, Bigras et Zoccolillo (2004), la consistance interne des échelles, évaluée par les indices a de Cronbach, est excellente et varie entre 0,79 et 0,94 .

L'échelle d'environnement familial (Moos \& Moos, 1986, Moos, 1989; 27 items) est une version française et abrégée du Family Environment Scale. Ses items, suivis d'un choix "vrai ou faux ", sont regroupés en trois sous-échelles d'égale longueur : cohésion familiale, expression affective par les membres de la famille et conflits intrafamiliaux. Pour chacune d'elles, les résultats bruts sont les totaux de "vrais ". À défaut de score T, une division par le maximum possible de 9 permet de convertir les scores bruts en indices décimaux variant entre 0 et 1 . L'indice de consistance interne de la version anglaise varie de 0,79 à 0,86 pour chaque sous-échelles.

Le questionnaire portant sur les pratiques éducatives (Frick, 1996; 36 items) est la version française de l'Alabama Parenting Questionnaire. Ses questions, suivies d'une échelle Likert en cinq points, évaluent : l'implication parentale dans l'éducation (10 items), le manque de supervision parentale ( 7 items), les comportements parentaux positifs (4 items), l'incohérence disciplinaire (5 items), le recours aux punitions corporelles (3 items) et les mesures disciplinaires spécifiques (au nombre de 7, ex. : privation de sorties). Les résultats bruts sont les totaux de " vrais " pour chacune des sous-échelles. À défaut de score T, une division du score brut par le nombre d'items permet de retrouver la réponse la plus souvent choisie. Selon Pauzé et al (2004), les indices a de Cronbach varient de 0,57 à 0,74 pour la version française.

L'échelle d'empowerment dans une famille (Koren, DeChillo, \& Friesen, 1992; 12 items), traduite du Family Empowerment Scale, évalue les sentiments de pouvoir et de contrôle des parents. Elle est composée de 12 items suivis d'une échelle Likert allant de 1 à 5 . Plus la valeur est élevée, meilleur est le sentiment d'empowerment. L'indice de consistance interne de la version française est de 0,88. 
Pour l'ensemble des variables issues de ces instruments, des analyses univariées et bivariées (t-test, chi carré) ont permis de comparer le groupe de jeunes ou de parents suivis dans un contexte de non-judiciarisation à celui des jeunes contrevenants suivis en vertu de la LSJPA ou de leurs parents. Lorsque le postulat d'homogénéité des variances n'est pas respecté, un test non paramétrique a été choisi. Le seuil de signification a été fixé à 0,05.

\section{Présentation des résultats}

\section{Les adolescents}

Profil des participants. Tous les jeunes interrogés sont des garçons, âgés en moyenne de 14 ans 10 mois. Aucune différence significative n'est mesurée entre les deux groupes (14 ans 6 mois versus 15 ans 1 mois). Ils reconnaissent avoir manifesté leurs premiers TCptSx entre 11 et 16 ans.

Au Questionnaire portant sur les distorsions cognitives, le score moyen pour l'ensemble des jeunes est de 3.0. Lorsque ce résultat est divisé par le maximum de 32 , l'indice décimal prend la valeur de 0,10. Les jeunes auteurs d'agression sexuelle rapportent donc très peu de distorsions telles que « mon frère ou ma sœur ne diront à personne que nous avons des relations sexuelles parce qu'ils aiment vraiment ça ». Les distorsions les plus souvent jugées vraies sont « certaines personnes sont gênées de demander d'avoir des relations sexuelles alors elles veulent vraiment qu'on les force » et " un très jeune enfant est capable de prendre la décision s'il veut ou non avoir des relations sexuelles avec moi ». Par ailleurs, on n'observe aucune différence statistiquement appréciable entre les adolescents non judiciarisés (moy= 3,1 ) et judiciarisés (moy $=2,8$ ).

À l'échelle d'attribution de la responsabilité, les 47 AAAS paraissent assumer une part significative de responsabilité (ex. : " j'ai très honte du (des) geste (s) que j'ai posé(s) » ou « j'aimerais beaucoup réparer les torts que j'ai faits »). Le résultat moyen pour la sous-échelle du sentiment de culpabilité est de 14,50/18, l'indice décimal valant 0,79 . À la sous-échelle d'attribution à un état mental perturbé, la moyenne est de 6,18/9 (indice décimal de 0,69; ex. : " je ne me reconnais pas moi-même dans le(s) geste(s) que j'ai posé(s) " ou " c'était plus fort que moi »). Quant à la moyenne pour la sous-échelle d'attributions externes, elle est de 2,25/15 (indice décimal de 0,15; ex. : " selon moi, la victime était grandement à blâmer » ou " j'avais de très bonnes raisons de poser le(s) geste(s) que j'ai posé(s) »). Aucune différence significative n'est mesurée entre les deux groupes. Tous les jeunes sont portés à assumer la responsabilité de leur agression sexuelle, même s'ils ont aussi tendance à invoquer un état mental anormal au moment du passage à l'acte.

À l'Inventaire de personnalité Jesness, l'ensemble des AAAS obtient des scores $T$ cliniquement élevés $(\geq 60)$ aux échelles d'immaturité $(63,7)$ et de mésadaptation sociale $(63,4)$. La valeur moyenne de l'indice d'asocialité $(61,3)$ est suffisamment élevée pour que les risques de mésadaptation soient significatifs. Aucune échelle ne permet de distinguer les jeunes qui présentent des TCptSx de ceux, judiciarisés, qui font appel à des organismes d'intervention psychosociale. 
Tableau 1. Comparaison entre les jeunes qui consultent dans les milieux médicaux et les contrevenants pris en charge en vertu de la LSJPA

\begin{tabular}{lccc}
\hline & Moyenne (E-T) & Test \\
\hline Total & Adolescents & Jeunes \\
$(47)$ & non judiciarisés & contrevenants \\
$(\mathrm{n}=30)$ & $(\mathrm{n}=17)$ \\
\hline
\end{tabular}

Le questionnaire des distorsions cognitives pour adolescents

Échelle de distorsions cognitives $3,04(2,6) \quad 3,12(2,9)$

$2,83(2,3) \quad 0,37$

L'échelle d'attribution de la responsabilité

Sentiment de culpabilité

$14,29(2,5)$

$14,52(2,6)$

$13,74(2,4) \quad 1,12$

Attribution à un état mental perturbé

$6,18(1,9)$

$6,35(1,7)$

$5,74(2,1)$

1,21

Attributions externes

$2,25(1,8)$

$2,31(1,6)$

$2,06(1,9)$

0,51

Inventaire de personnalité Jesness (Score T)

Immaturité

Mésadaptation sociale

Autisme

Orientation des valeurs

Aliénation

Agressivité manifeste

Refoulement

Retrait

Anxiété sociale

Déni

Indice d'asocialité
$63,59^{\#}(5,5)$

$63,66^{\#}(4,3)$

$63,41^{\#}(6,6)$

0,10

$63,07^{\#}(12,8)$

$63,36^{\#}(12,0)$

$62,35^{\#}(13,1)$

0,28

$59,26(8,7)$

$60,18^{\#}(9,1)$

$56,88(8,5)$

1,28

$56,69(9.8)$

$57,29(8,6)$

$55,12(10,5)$

0,83

$56,40(8,6)$

$56,61(8,7)$

$55,82(8,5)$

0,32

$55,27(9.4)$

$55,96(7,5)$

$53,47(11,2)$

0,84

$55,13(13,0)$

$54,14(11,5)$

$57,65(14,5)$

$-0,98$

$54,76(9,9)$

$54,89(9,4)$

$54,41(11,0)$

0,17

$53,23(9,5)$

$54,00(7,7)$

$51,18(11,3)$

1,12

$43,43(10,0)$

$42,51(9,7)$

$45,76(10,2)$

$-1,15$

$61,30^{\#}(12,8)$

$60,90 *(12,9)$

$62,24^{\#}(12,7) \quad-0,36$

Échelle des symptômes traumatiques pour enfants (Score T)

Préoccupations sexuelles

Dépression

$62,62^{\#}(15,8) \quad 63,94^{\#}(15,0)$

$58,88(16,7) \quad 1,16$

Anxiété

$61,26^{*}(12,8) \quad 63,04^{*}(13,6)$

$56,24(12,1) \quad 1,81^{*}$

Stress post-traumatique

$59,75(13,2) \quad 61,85^{*}(14,4)$

$53,82(11,1) \quad 2,08^{* *}$

Dissociation

$59,09(11,2) \quad 60,29^{\#}(11,4)$

$55,71(11,1) \quad 1,49$

$58,38(11,5) \quad 60,13^{\#}(13,1)$

$53,53(9,6) \quad 1,89^{*}$

Tendance à exagérer

$58,38(17,0)$

$59,78(21,7)$

$53,8811,7)$

1,10

Colère

$49,72(7,9)$

$50,29(7,9)$

$48,12(7,9)$

0,97

Tendance à minimiser

$47,68(8,9)$

$47,42(8,9)$

$48,41(8,9) \quad-0,39$

L'échelle d'attitudes face au père et face à la mère

Attitude de l'enfant face au père $\quad 30,00(25,1) \quad 29,83(24,3) \quad 30,54(26,9) \quad-0,09$

Attitude de l'enfant face à la mère

$25,83(17,3) \quad 24,96(17,9) \quad 28,17(16,8) \quad-0,66$

\# Résultat se situant au-delà d'un seuil clinique; * $p \leq .05 ;{ }^{* *} p \leq .01$ 
Sous l'angle des symptômes traumatiques pour enfants, les échelles de validité indiquent d'abord que les résultats obtenus sont fiables, quoiqu'une légère tendance à exagérer la symptomatologie puisse être notée (score $T=58,4)$. Dans l'ensemble, les 47 participants présentent un niveau élevé $(\geq 60)$ de préoccupations sexuelles (ex. : "penser au sexe quand je ne le veux pas " ou "ressentir des sensations sexuelles dans mon corps " $(62,6)$ et de symptômes dépressifs tels qu'« avoir l'impression d'avoir fait quelque chose de mal » $(61,2)$. Sous cet angle, des différences significatives sont notées : les jeunes présentant des TCptSx rapportent un niveau plus élevé de symptômes dépressifs ( 63,9 c. 58,9, $p<0,05)$, anxieux (ex. : " me sentir nerveux ou agité à l'intérieur »; 61,9 c. 53,8, p<0,01) et dissociatifs (ex: "rêvasser ou être perdu dans ses pensées, être dans la lune »; $60,1$ c. $53,5, p<0,05)$ que les adolescents judiciarisés.

Sous l'angle des attitudes face au père et face à la mère, globalement, les AAAS mentionnent avoir une bonne relation avec leurs deux parents, quoique meilleure avec leur mère (moy $=25,8$ ) qu'avec leur père (moy : 30,0; ex. : " mon père est trop exigeant » ou il " se mêle de mes activités »). Entre les deux groupes, aucune différence significative ne peut être détectée.

Enfin, à partir des réponses des jeunes, il a été possible de calculer un indice de statut socio-économique. Au tableau 2, les résultats montrent que la majorité des participants décrivent l'âge, le niveau de formation et la catégorie professionnelle d'un de leurs parents de telle sorte que leur statut peut être qualifié de moyen (52\% et $53 \%$ ) ou moyen défavorisé ( $23 \%$ et $27 \%)$. Aucune différence significative ne peut être mesurée à ce niveau.

Tableau 2. Statut socioéconomique selon les informations rapportées par le jeune

\begin{tabular}{lcc}
\hline Indice de position socioéconomique & $\begin{array}{c}\text { Adolescents non } \\
\text { judiciarisés } \\
\mathrm{n}(\%)\end{array}$ & $\begin{array}{c}\text { Jeunes contrevenants } \\
\mathrm{n}(\%)\end{array}$ \\
\hline Défavorisé (1-35) & $3(10)$ & $2(11)$ \\
Moyen-défavorisé (36-54) & $8(27)$ & $4(23)$ \\
Moyen (55-67) & $16(53)$ & $9(52)$ \\
Moyen favorisé (68-80) & $1(3)$ & $1(5)$ \\
Favorisé (+ de 80) & $2(7)$ & $1(5)$ \\
\hline
\end{tabular}




\section{Les parents}

Rappelons que les parents participants à l'étude sont répartis en deux groupes : 20 ont un adolescent suivi dans un contexte de non-judiciarisation, six ont un jeune judiciarisé et encadré par la LSJPA. Les caractéristiques de ces parents sont présentées aux tableaux 3 et 4 .

Tableau 3. Antécédents de consultation antérieure des parents à propos des problèmes émotionnels ou comportementaux du jeune

\begin{tabular}{|c|c|c|c|}
\hline \multirow{2}{*}{$\begin{array}{l}\text { Type de consultation antérieure à la } \\
\text { demande actuelle }\end{array}$} & \multicolumn{3}{|c|}{ Nombre (et pourcentage) } \\
\hline & $\begin{array}{c}\text { Total } \\
(n=26)\end{array}$ & $\begin{array}{c}\text { Parents } \\
\text { d'adolescent } \\
\text { non } \\
\text { judiciarisé } \\
(n=20)\end{array}$ & $\begin{array}{c}\text { Parents } \\
\text { de jeune } \\
\text { contrevenant } \\
(n=6)\end{array}$ \\
\hline Centre d'écoute téléphonique & $10(38)$ & $7(35)$ & $3(50)$ \\
\hline Travailleur social à l'extérieur de l'école & $8(31)$ & $5(25)$ & $3(50)$ \\
\hline Centre jeunesse & $7(27)$ & $6(30)$ & $1(17)$ \\
\hline $\begin{array}{l}\text { Bureau privé d'un professionnel de la } \\
\text { santé }\end{array}$ & $6(23)$ & $4(20)$ & $2(33)$ \\
\hline $\begin{array}{l}\text { Pédiatre, omnipraticien ou médecin de } \\
\text { famille }\end{array}$ & $6(23)$ & $4(20)$ & $2(33)$ \\
\hline $\begin{array}{l}\text { Professionnel spécialisé pour des } \\
\text { problèmes d'apprentissage ou de } \\
\text { développement à l'extérieur de l'école }\end{array}$ & $6(23)$ & $5(25)$ & $1(17)$ \\
\hline $\begin{array}{l}\text { Organisme communautaire pour les } \\
\text { jeunes }\end{array}$ & $5(19)$ & $4(20)$ & $1(17)$ \\
\hline $\begin{array}{l}\text { Urgence, clinique ou service externe } \\
\text { d'un hôpital }\end{array}$ & $4(15)$ & $3(15)$ & $1(17)$ \\
\hline Psychiatre ou psychologue & $2(8)$ & $2(10)$ & $0(0)$ \\
\hline $\begin{array}{l}\text { Centre local de services } \\
\text { communautaires (CLSC) }\end{array}$ & $2(8)$ & $2(10)$ & $0(0)$ \\
\hline
\end{tabular}

Par rapport à la trajectoire de consultations antérieures, les participants ont indiqué s'ils avaient déjà demandé de l'aide en raison d'éventuels problèmes émotionnels ou comportementaux de leur fils. Globalement, $77 \%$ des parents identifient au moins un service. De ce nombre, $15 \%$ ont eu recours à un type de services et $11 \%$ à deux types. Le plus grand nombre de services auxquels un même parent a fait appel est cinq. Ces mères et pères biologiques se sont surtout tournés vers des centres d'écoute téléphonique (38\%), des travailleurs sociaux $(31 \%)$ et des intervenants en centre jeunesse (27\%). Aucune différence ne distingue les deux groupes à ce niveau, un test de chi carré étant non concluant. 
Pour l'ensemble des souvenirs traumatiques de l'enfance, le total brut est de 117. Pour les parents, la réponse 2 (rarement) est la plus fréquente. Sous l'angle de la négligence émotionnelle (ex. : " mon père était rarement présent à la maison » ou " j'ai rarement eu l'amour et l'attention dont j'avais besoin »), le total de 49,6 indique que les parents ont régulièrement choisi la réponse 3 (" quelques fois »). Au niveau de l'abus physique et émotionnel (ex. : " un membre de ma famille hurlait ou criait après moi »), le total de 44,4 signifie que les participants ont généralement inscrit 2 (« rarement »). Pour la négligence physique (ex. : « j'ai dû porter des vêtements sales. "), le total de 16,05 traduit plusieurs réponses 1 (" jamais »). Enfin, par rapport à l'abus sexuel (ex. : " quelqu'un a essayé de me faire poser des gestes sexuels ou de me faire voir des choses sexuelles. "), le total de 7,6 indique que les parents ont surtout répondu 1 (" jamais »). Aucune différence significative ne sépare les groupes, même si les parents de jeunes contrevenants ont une légère tendance $(p<0,10)$ à rapporter plus de souvenirs de traumatismes sexuels.

Selon Moos (1989), la cohésion familiale se définit par le degré d'engagement, d'aide et de support entre les membres. À cette échelle, le résultat se situe normalement entre 6 et 7 (sur un total de 9). Le score moyen obtenu pour l'ensemble des 26 parents est de 7,15, donc comparable à la norme. Sous l'angle de l'expression, c'est-à-dire de la tendance à agir ouvertement et à exprimer ses sentiments dans la famille, l'instrument de Moos produit des résultats qui se situent communément entre 5 et $6(\max =9)$. Dans cette étude, la moyenne est de 5,73. À l'échelle de conflits, qui renvoie à l'expression de colère, d'agressivité, de disputes et de critiques envers les membres de la famille, en général les cotes varient de 2,5 à $4(\max =9)$. Dans cette étude, le score de 6,56 signifie que ces parents rapportent vivre plus de conflits (ex : " les membres de ma famille se critiquent souvent les uns les autres »). Enfin, du point de vue des parents, aucune différence significative ne sépare l'environnement familial des adolescents judiciarisés et non judiciarisés.

Par rapport aux pratiques éducatives, dans le tableau 3 on constate que les 26 parents de l'échantillon estiment faire preuve d'une relative implication (ex. : « vous vous informez de la journée qu'il a passée à l'école »). Le score brut moyen de 32,22 correspond à une majorité de réponses 3 (" quelques fois»). Vient ensuite l'échelle des manifestations de comportements positifs (ex. : " vous lui soulignez ses réalisations et ses points positifs ») où le résultat moyen est de 12,50. La réponse la plus fréquente est donc 3 ou « quelques fois ». Peu d'incohérences disciplinaires (ex. : « les punitions que vous lui donnez dépendent de votre humeur ») et d'omissions dans la supervision parentale (ex. : " il est à l'extérieur tard la nuit sans surveillance ») sont rapportées. De ce point de vue, les moyennes de 13,35 et 14,78 correspondent aux libellés « quelques fois » et « presque jamais ». II semble que le recours aux punitions corporelles (ex: "vous le frappez avec la main ou le giflez lorsqu'il fait quelque chose de mal ") soit encore plus rare : le total de 3,43 signifie que la majorité des participants répondent " jamais ». Enfin, soulignons qu'aucune différence n'est observée entre les deux échantillons.

Du point de vue de l'empowerment, l'ensemble des parents d'AAAS obtient un résultat moyen de 51,12 (ou 4,2 par item). Des énoncés tels que « je suis capable d'aller chercher de l'information pour m'aider à mieux comprendre mon fils » ont 
Tableau 4 Comparaison entre les parents d'auteurs de TCptSx qui consultent dans les milieux médicaux et de contrevenants pris en charge en vertu de la LSJPA

\begin{tabular}{cccc}
\hline & Moyenne $(\mathrm{E}-\mathrm{T})$ & Test $\mathrm{t}$ \\
\hline Total & Parents & Jeunes \\
$(\mathrm{n}=26)$ & d'adolescent & contrevenants \\
& non & $(\mathrm{n}=17)$ \\
& judiciarisé & \\
& $(\mathrm{n}=20)$ & \\
& & \\
\end{tabular}

Le questionnaire portant sur les souvenirs traumatiques de l'enfance

Négligence émotionnelle $\quad 49,60(18,3) \quad 48,09(19,5) \quad 56,00(14,6) \quad-0,75$

Abus physique et émotionnel

$44,40(21,2) \quad 43,15(22,2) \quad 49,75(18,1) \quad-0,55$

Négligence physique

$16,05(8,8) \quad 16,06(9,9) \quad 16,00(5,1) \quad 0,01$

Abus sexuel

$7,64(4,5)$

$6,79(4,0)$

$11,25(6,1)$

$-1,79^{+}$

Score total de souvenirs traumatiques

$117,69(50,4) \quad 114,09(53,2) \quad 133,00(41,3) \quad-0,66$

\begin{tabular}{|c|c|c|c|c|}
\hline \multicolumn{5}{|c|}{ L'échelle d'environnement familial } \\
\hline Cohésion & $7,15(2,0)$ & $7,08(2,0)$ & $7,50(1,9)$ & $-0,39$ \\
\hline Expression & $5,73(1,2)$ & $5,83(1,1)$ & $5,25(1,7)$ & 0,83 \\
\hline Conflit & $6,56(2,3)$ & $6,73(2,3)$ & $5,75(2,2)$ & 0,76 \\
\hline \multicolumn{5}{|c|}{ Le questionnaire portant sur les pratiques éducatives } \\
\hline Implication parentale & $32,26(2,7)$ & $32,35(2,8)$ & $31,67(2,3)$ & 0,40 \\
\hline $\begin{array}{l}\text { Comportements } \\
\text { parentaux positifs }\end{array}$ & $12,50(1,0)$ & $12,53(1,2)$ & $12,33(0,5)$ & 0,26 \\
\hline Incohérence disciplinaire & $13,35(2,5)$ & $13,35(2,9)$ & $13,33(1,1)$ & 0,01 \\
\hline $\begin{array}{l}\text { Manque de supervision } \\
\text { parentale }\end{array}$ & $14,78(3,4)$ & $14,75(3,1)$ & $15,00(4,3)$ & $-0,12$ \\
\hline Punition corporelle & $3,43(0,6)$ & $3,35(0,5)$ & $4,00(1,7)$ & $\begin{array}{r}Z^{(a)} \\
-0,45\end{array}$ \\
\hline $\begin{array}{l}\text { Mesures de discipline } \\
\text { spécifiques }\end{array}$ & $19,87(2,3)$ & $19,75(2,6)$ & $20,67(1,5)$ & $-0,58$ \\
\hline \multicolumn{5}{|c|}{ L'échelle d'empowerment dans une famille } \\
\hline Pouvoir d'agir & $51,12(4,7)$ & $51,84(6.0)$ & $48,00(0,0)$ & $\begin{array}{l}Z^{(a)} \\
-1,17\end{array}$ \\
\hline
\end{tabular}

$\dagger: p \leq .10$; (a) L'égalité des variances n'étant pas assumée pour ces variables, le test de Levene a été remplacé par le test non paramétrique de Mann-Whitney. 
donc souvent été jugés « assez vrais ». Autrement dit, les participants estiment gérer suffisamment bien la situation, que ce soit en se renseignant ou en posant des actions. Aucune différence significative ne peut être repérée entre les deux groupes.

\section{Discussion des résultats}

À notre connaissance, cette recherche est l'une des premières à s'intéresser spécifiquement à la situation des AAAS non judiciarisés au Québec. Dans un premier temps, il s'agissait de comparer à l'aide de six questionnaires le portrait d'auteurs de TCptSx et celui de jeunes contrevenants judiciarisés et encadrés en vertu de la LJSPA. Peu de différences ont été notées.

En 1997, dans une étude visant à valider la version française du questionnaire portant sur les distorsions cognitives, Madrigrano, Robinson et Rouleau obtenaient le score brut moyen de 2,6 auprès d'un échantillon d'adolescents normaux et 3,0 pour les AAAS. Ceci amenait les auteurs à considérer que les jeunes avaient « coté faiblement » (p. 10) à l'instrument. Nos résultats vont dans le même sens.

Après avoir utilisé l'échelle d'attribution de la responsabilité de Gudjonsson auprès de criminels adultes, Kroger et Mills (2004) ont présenté les statistiques suivantes : pour la sous-échelle de sentiment de culpabilité une moyenne de 11,7, comparativement à 2,8 pour les attributions externes et 4,3 pour les attributions à un état mental perturbé. Par comparaison, les AAAS évalués dans cette étude rapportent se sentir plus coupables $(14,50)$ et sont plus portés à blâmer la perturbation de leur état mental au moment de l'agression $(6,18)$. La faible tendance à attribuer la responsabilité de son comportement à l'environnement extérieur correspond à la présence négligeable de distorsions cognitives mentionnée dans le paragraphe précédent.

Au Jesness, les AAAS obtiennent des scores T cliniquement élevés aux échelles d'immaturité, de mésadaptation sociale et à l'indice global d'asocialité. Selon le manuel de cet instrument, les participants dits " immatures " sont naïfs dans l'évaluation de leurs motivations et celles d'autrui, ils répriment leurs problèmes pour se conformer aux normes et gardent une position passive dans leurs relations interpersonnelles. Quant à la mésadaptation sociale, elle désigne ici des attitudes associées à une mauvaise intégration à la vie collective : une représentation négative de soi, une propension à blâmer autrui pour ses propres difficultés, un faible contrôle sur l'agressivité, une hypersensibilité à la critique et un développement lacunaire du jugement moral. Bref, selon le profil obtenu au Jesness, les AAAS sont à risques de troubles d'adaptation en raison de leur immaturité et non pas de leur adhésion à des valeurs délinquantes ou de la présence d'une agressivité manifeste. Les jeunes contrevenants et ceux qui présentent des TCptSx ne se distinguent pas sous cet angle.

Par ailleurs, les AAAS rapportent avoir une bonne relation avec leurs deux parents, quoique meilleure avec leur mère. 
Entre les jeunes non judiciarisés et ceux qui sont judiciarisés, une seule différence significative a pu être mesurée, mais elle a son intérêt : les jeunes qui consultent en milieux médicaux rapportent des niveaux plus élevés de symptômes dépressifs, anxieux et dissociatifs (ex : "rêvasser ou être perdu dans ses pensées, être dans la lune ») qui pourraient être consécutifs à des antécédents traumatiques.

De ces résultats, quelques pistes d'intervention peuvent être déduites. Tout d'abord, dans l'ensemble, les jeunes qui ont participé à l'étude ne rapportent que peu de distorsions cognitives, attributions externes du blâme, valeurs délinquantes et conflits ouverts avec leurs parents. On pourrait évidemment se demander si, par désirabilité sociale, ils n'ont pas eu tendance à nier et minimiser certains de ces problèmes. Par rapport à cette hypothèse, il faut tout de même signaler que l'inventaire de Jesness n'a détecté aucune propension significative au déni (score $T=43,4$ ), ni à la répression (score $T=55,1$ ). Si nos résultats ne sont pas entachés de désirabilité sociale, alors ils indiquent que la confrontation de croyances propices aux agressions, les interventions centrées sur la responsabilisation ou la restructuration des distorsions cognitives ne sont pas toujours nécessaires. Par contre, un soin particulier devrait être mis à recadrer a) la notion de consentement, telle qu'elle est comprise par les AAAS et b) leur tendance marquée à attribuer la cause du passage à l'acte à un état mental perturbé.

Auprès des jeunes dont la situation n'est pas judiciarisée plus spécifiquement, un dépistage et une évaluation des symptômes anxio-dépressifs, de même que des préoccupations sexuelles d'origine post-traumatique semblent indiqués.

Dans un deuxième temps, il s'agissait de comparer, à l'aide de cinq questionnaires, les parents des jeunes dont la prise en charge est non judiciarisée et ceux dont l'adolescent est encadré par la LJSPA. Rappelons d'abord que c'est souvent la mère biologique qui a rempli les questionnaires de la recherche comme c'est le cas dans la plupart des études portant sur des jeunes en difficulté. Le taux élevé de refus, particulièrement chez les parents de contrevenants encadrés par la LSJPA, limite évidemment à ce point-ci les possibilités de généraliser les conclusions à l'ensemble des familles.

Les résultats montrent néanmoins que la plupart des parents ont déjà eu recours à au moins un service de santé ou service psychosocial (ex. : centres d'écoute téléphonique, centres jeunesse, professionnels de la santé) pour des problèmes émotifs ou comportementaux manifestés par le jeune. On peut affirmer que ces jeunes et leurs parents ont déjà une trajectoire de consultations bien engagée. Il serait intéressant de s'y pencher davantage, dans une recherche future. En effet, cette trajectoire pourrait aider à mieux comprendre pourquoi certains AAAS voient leur situation être judiciarisée et d'autres pas.

Dans l'ensemble des questionnaires, très peu de données permettent de différencier les parents d'adolescents qui font appel aux milieux médicaux des autres. C'est au niveau des souvenirs traumatiques que se trouve le seul indice distinctif. II tient au fait que les parents de jeunes contrevenants suivis en vertu de la LSJPA ont légèrement tendance $(p<0,10)$ à rapporter plus de souvenirs personnels 
de traumatismes sexuels vécus durant l'enfance. II est évident qu'un plus grand échantillon serait requis pour pousser plus loin la réflexion à ce niveau. On peut néanmoins faire prudemment l'hypothèse selon laquelle des parents sexuellement victimisés ont plus de mal à gérer par eux-mêmes le dévoilement de l'agression. Les policiers et le système de justice sont alors plus amenés à intervenir.

Par rapport à la perception de l'environnement familial, dans l'étude de Busconi (et al., 1998), les parents d'AAAS ont rapporté moins de conflits intrafamiliaux (ex. : "les membres de ma famille se critiquent souvent les uns les autres »; moy $=4,13$ ) que les 26 parents de notre échantillon. Nos propres résultats corroborent plutôt ceux de Ford et Linney (1995) qui suggèrent la relative importance des conflits dans ces familles.

Pour mieux juger du portrait des pratiques parentales ici tracé, il faut d'abord préciser qu'à l'Inventaire Alabama, un score de 3,25 ou moins indique la présence d'un problème quant à l'engagement parental et aux pratiques parentales positives selon Pauzé et ses collaborateurs (2004). Pour les échelles du manque de supervision et de la discipline inconsistante, un score de 2,5 et plus est considéré comme problématique. Dans notre étude, les scores obtenus aux indices d'implication, manifestation de comportements positifs, et incohérences se situent à la limite de ces seuils cliniques. Cela semble indiquer que ces adultes ne croient pas avoir de problèmes majeurs sur le plan des pratiques éducatives, même s'ils rapportent de légères difficultés. II n'est donc pas étonnant de constater que, dans l'ensemble, les participants considèrent pouvoir gérer assez bien la situation de leur fils.

Étant donné les efforts qu'il a fallu déployer pour recruter les parents d'AAAS dans cette étude, il y a lieu de penser que les intervenants ont tout un défi à relever lorsque vient le temps de leur proposer un accompagnement clinique. Nous avons indiqué ailleurs qu'au Québec, la plupart des intervenants estiment qu'une action auprès des parents est nécessaire, mais que dans les faits elle est très rarement implantée sur une base régulière (Lafortune, Tourigny, Proulx, \& Metz, 2007). Les résultats obtenus ici permettent de conclure que des besoins semblent néanmoins présents sur le plan de l'apprentissage de stratégies de résolution des problèmes et des conflits familiaux. Cela pourrait être une manière intéressante d'approcher les familles qui se présentent en consultation.

Pour conclure, il appert que les jeunes dont la situation n'a pas été judiciarisée ressemblent beaucoup à ceux qui sont pris en charge en vertu de la LSJPA, que ce soit en termes de leur âge, du statut socioéconomique du parent le plus significatif dans leur vie, de leur immaturité et de leur risque de mésadaptation sociale. Un élément semble les distinguer et il s'agit de la présence plus élevée de symptômes de stress post-traumatiques. Sur le plan familial, des résultats très préliminaires indiquent que les familles des jeunes judiciarisés et non judiciarisés se ressemblent aussi. La seule spécificité tient au fait que les parents des jeunes non judiciarisés ont tendance à rapporter un peu moins de souvenirs de traumatismes sexuels que les parents de l'autre groupe. Une question se pose : se peut-il que la prévalence des symptômes de stress post-traumatiques chez les jeunes favorise la non-judiciarisation de la prise en charge tandis que la prévalence des mêmes symptômes chez un des parents favorise le déclenchement de procédures légales? 


\section{Références}

Becker, J.V. \& Kaplan, M.S. (1988). The assessment of adolescent sexual offenders. Advances in behavioral assessment of children and families, 4 , 97-118.

Bengis, S. (1997). Comprehensive service delivery with a continuum of care. In G. Ryan, et S. Lane (Eds.), Juvenile sexual offending (pp. 211-218). San Francisco, CA: Jossey-Bass.

Bernstein, D.P., Fink, L., Foote, J., Handelsman, L. et al., (1994). Initial Reliability and Validity of a New Retrospestive Mesure of Child Abuse and Neglect. American Journal of Psychiatry, 151, 18,1132-1136.

Blaske, D.M., Borduin, C.M., Henggeler, S.W., \& Mann, B.J. (1989). Individual, familiy and peer characteristics of adolescent sex offenders and assaultive offenders. Developmental Psychology, 25, (5), 846-855.

Born, M. \& Thys, P. (2001). Délinquance juvénile et famille. Paris, France : L'Harmattan

Briere, J. (1989). The trauma symptom checklist for children (TSCC) (Unpublished test): University of Southern California School of Medecine.

Burton, D.L., Miller, D.L., \& Chill, C.T. (2002). A social learning theory comparison of the sexual victimization of adolescent sexual offenders and nonsexual offending male delinquents. Child Abuse \& Neglect, 26, (9), 893-907

Busconi, A., Gray, A., Houchens, P., \& Pithers, W.D. (1998). Caregivers of Children with Sexual Behavior Problems: Psychological and Familial Functioning. Child Abuse and Neglect, 22, 12, 129-141

Carpentier. J., Leclerc, B., \& Proulx, J. (2011). Juvenile sexual offenders: Correlates on onset, variety and desistance of criminal behavior. Criminal Justice and Behavior, 38, 854-873.

Comeau, S. \& Boisvert, J.M. (1985). Un ensemble de mesures cliniques. Document inédit
Dozois, J. (1994). Adolescent et agresseur sexuel: bilan d'une recherche. Criminologie, 27, 2, 71-85.

Dufort, F. \& Le Bossé, Y. (1999). Le pouvoir d'agir (empowerment) des personnes et des communautés : une autre façon d'intervenir. Chicoutimi/SainteFoy, Gaëtan Morin/Les Presses de I'Université Laval.

Epstein, N.B., Baldwin, L.M., \& Bishop, D.S. (1983). The McMaster family assessment device. Journal of Marital and Family Therapy, 9, 171-180.

Ford, M.E. \& Linney, J.A. 1995. Comparaison analysis of juvenile sexual offenders, violent nonsexual offenders, and status offenders. Journal of Interpersonal Violence, 10, 11, p. 56-70.

Frick, P.J. (1996). Assessment of Parenting Practices in Families of Elementary School-Age Children. Journal of Clinical child Psychology, 25, 13, 317-329.

Gaudreau, L. (1991). L'éducation sexuelle, l'agression sexuelle et la prévention chez les enfants et les adolescents in: $\mathrm{H}$. Cohen (Ed.): L'agression sexuelle, perspectives contemporaines (pp.175210). Montréal : Editions du Méridien

Genoud, P.A. (2011). Indice de position socioéconomique : un calcul simplifié. Fribourg : Université de Fribourg. Document non publié

Gudjonsson, G.H. \& Singh, K.K. 1989. The Revised Gudjonsson Blame Attribution Inventory. Personnality Individual Differences, 10, 11, 6770.

Giuli, C.A., \& Hudson, W.W. (1977). Assessing parent-child relationship disorders in clinical practice: The child's point of view. Journal of Social Service Research, I (11), 77-92.

Jacob, M., McKibben, A., \& Proulx, J. (1993). Étude descriptive et comparative d'une population d'auteurs de comportements sexuels problématiques. Criminologie, 26, 1, 13-163. 
Jesness, C.F. (1983). The Jesness Inventory manual. Palo Alto: Consulting Psychologists Press.

Johnson, T.C. (1988). Child perpetrators: children who molest other children, preliminary findings. Child Abuse and Neglect, 12, 219-229.

Jonson-Reid, M. \& Way, I. (2001). Adolescent sexual offenders compared to other incarcerated delinquents: Childhood maltreatment, serious emotional disturbance, and offending histories. American Journal of Orthopsychiatry, 71 (1), 1-11.

Kaplan, M.S., Becker, J. V., \& CunninghamRathner, J. (1988). Characteristics of parents of adolescent incest perpetrators: Preliminary findings. Journal of Family Violence, 3, 3, 183191.

Kaplan, M.S., Becker, J.V., \& Martinez, D.F. (1990). A comparison of mothers of adolescent incest vs non-incest perpetrators. Journal of Family Violence, 5, 3, 209-214.

Kavoussi, R.J., Kaplan, M. S., \& Becker, J.V. (1988). Psychiatric diagnosis in adolescent sex offenders. Journal of the American Academy of Child and Adolescent Psychiatry, 27, 241-243.

Knopp, F.H. (1985). Remedial interventions in adolescent sex offenses: Nine program descriptions. Syracuse: Safer Society Press.

Koren, P.E., De Chillo, N., \& Friesen, B.J. (1992). Measuring Empowerment in Families Whose Children Have Emotional Disabilities: a Brief Questionnaire. Rehabilitation Psychology, 37, 305-321.

Kroger, D.G. \& Mills, J.F. (2004) The Criminal Attribution Inventory: A Measure of Offender Perceptions. Journal of Offender Rehabilitation, 39, 4, 15-29.

Lafortune, D. (1996). Antécédents et caractéristiques psychologiques de l'adolescent ayant commis un abus sexuel. Thèse de doctorat inédite, Université de Montréal
Lafortune, D. (2001). Abus sexuel juvénile et délinquance juvénile à la lumière des antécédents personnels et familiaux. Revue internationale de criminologie et de police technique et scientifique, 3 , 299-317.

Lafortune, D., Tourigny, M., Proulx, J., \& Metz, K. (2007). Les interventions pour adolescents agresseurs sexuels implantées au Québec. Revue Canadienne de Psychoéducation, 36, 1, 25-56.

Lafortune, D. (2009). Rapport final de l'étude exploratoire des interventions offertes dans les milieux médicaux aux jeunes auteurs de comportements sexuels problématiques. Rapport remis au Centre national de prévention du crime et au Ministère de la Sécurité publique. Montréal : Centre International de Criminologie Comparée.

Lagueux, F. (2006). Évaluation des effets d'un programme de traitement de groupe pour adolescents agresseurs sexuels. Thèse de doctorat non publiée. Montréal : Université de Montréal.

Lagueux, F. \& Tourigny, M. (1999). État des connaissances au participant des adolescents agresseurs sexuel. Québec : ministère de la Santé et des Services sociaux.

Leblanc, M. \& Lapointe, C. (1999). Agression sexuelle chez les garçons : comparaison des agresseurs, des agressés et des agresseurs agressés avec les autres adolescents en difficulté. Revue canadienne de criminologie, 479-511.

Loeber, R., \& Farrington, D.P. (2000). Young children who commit crime : Epidemiology, developmental origins, risk factors, early interventions, and policy implications, Development and Psychopathology, 12, 737-762.

Longo, R.E. \& Groth, A.N. (1983). Juvenile sexual offenses in the histories of adult rapists and child molesters. International Journal of Offender Therapy and Comparative Criminology, 27,2, 150-155. 
Madrigrano, G.D., Robinson, M.C., \& Rouleau, J.L. (1997). Caractéristiques sociodémograpiques et cliniques d'une population d'adolescents ayant commis des crimes sexuels. Revue québécoise de psychologie, 18 (3) 91-110.

Moos, R.H. \& Moos, B.S. (1986). Family Environment Scale Manual (2nd ed.). Palo Alto, CA: Consulting Psychologists Press Inc.

Moos, R.H. (1989). Family Environment Scale-Form R, Interpretative Report Form. Palo Alto (Calif.), Consulting Psychologists Press Inc

Paivio, S.C., \& Cramer, K.M. (2004). Factor structure and reliability of the Childhood Trauma Questionnaire in a Canadian undergraduate student sample. Journal of Child Abuse and Neglect, 28, 889904.

Paquette, D., Laporte, L., Bigras, M., \& Zoccolillo, M. (2004). Validation de la version française du CTQ et prévalence de l'histoire de maltraitance. Santé mentale au Québec, 29, 1, 201-220

Pauzé, R., Centre Jeunesse de Montréal, \& Yergeau, R. (2004). Enfants, familles et parcours de services dans les centres jeunesse du Québec : portrait des jeunes âgés de 0 à 17 ans référés à la prise en charge des Centres Jeunesse du Québec (7 vol.). Sherbrooke : GRISE.
Rightland, S. \& Welch, C (2001). Juveniles who have sexually offended; A review of the professional literature. Washington D.C.: Office of Juvenile Justice and Delinquency Prevention.

Tardif, M., Jacob, M., Quenneville, R., \& Proulx, J. (2012). La délinquance sexuelle des mineurs: Approches cliniques. Montréal : Presses de l'Université de Montréal.

Thomas, J. (1991). The adolescent sex offender's family in treatment, Dans G. D. Ryan et S. L. lane (éds), Juvenile sexual offending: Causes, consequences and correction. Lexington (Mass.), Lexington Books, p. 334376

Vizard, E., Monck, E., \& Misch P. (1995). Child and adolescent sex abuse perpetrators: A review of the research literature. Journal of Child Psychology and Psychiatry. 36 (5), 731-756.

Vizard, E., Hickey, N., French, L., \& McCrory, E. (2007). Children and adolescents who present with sexually abusive behaviour: a UK descriptive study. Journal of Forensic Psychiatry and Psychology. 18:59-73

Worling, J.R. (1995). Adolescent SiblingIncest Offenders: Differences in Family and Individual Functioning When Compared to Adolescent Nonsibling Sex Offenders. Child Abuse and Neglect, 19, 15, p. 633-643. 